\title{
ON THE NUMBER OF ZEROS OF ANALYTIC FUNCTIONS IN A NEIGHBORHOOD OF A FUCHSIAN SINGULAR POINT WITH REAL SPECTRUM
}

\author{
M. Roitman And S. Yakovenko
}

\begin{abstract}
Consider a linear differential equation of some order $n$ with coefficients analytic in the unit disk. Assuming that the equation has a unique Fuchsian singular point at $z=0$, and all roots of the corresponding indicial equation are real, we establish an upper bound for the number of zeros of any solution of this equation in any sector with the vertex at $z=0$. This upper bound is in some sense linear in the magnitude of the coefficients of the equation.
\end{abstract}

\section{Introduction}

1.1. Zeros of analytic functions determined by linear ordinary differential equations: a brief survey. Let $f(t)$ be a function analytic on some subset $K \subset \mathbb{C}$ and known to satisfy a differential equation $\mathbf{L} f=0$ in a larger (open) domain $U \supseteq K$, where $\mathbf{L}$ is a linear ordinary differential operator of the form

$$
\mathbf{L}=a_{0}(t) \partial^{n}+a_{1}(t) \partial^{n-1}+\cdots+a_{n-1}(t) \partial+a_{n}(t), \quad \partial=\frac{d}{d t},
$$

with the coefficients $a_{j}(t)$ analytic and bounded in $U$. In this paper we address a particular case of the following general problem: find an upper bound for the number of isolated zeros of $f$ in $K$ in terms of the magnitude of the coefficients $a_{j}(t)$ in $U$. The latter notion apparently needs to be specified, since the coefficients $a_{j}(\cdot)$ can be simultaneously multiplied by a nonzero analytic function without changing the equation $\mathbf{L} f=0$.

This problem has already been investigated for various classes of equations and functions. If $\mathbf{L}$ is a second order operator with real coefficients, then upper bounds for the number of zeros can be obtained within the classical Sturm theory. The case of arbitrary $n$ and real zeros was studied in [1] in two different contexts. Let $K$ be a segment on the real line and $U$ an open neighborhood of $K$. If the operator $\mathbf{L}$ has no singular points in $U$

Received December 27, 1996.

1991 Mathematics Subject Classification Primary 34A20, secondary 58F14, 34C23

The second author is an incumbent of Elaine Blond Career development chair. The research was partially supported by the Minerva foundation. 
(i.e., the leading coefficient $a_{0}(t)$ is nonvanishing in $U$ ), then without loss of generality we may assume $a_{0}(t) \equiv 1$. The first main result of [1] asserts that in this case the number of zeros of any real solution of the equation $\mathbf{L} f=0$ on $K$ does not exceed $n+\gamma(\|\mathbf{L}\|+\ln n)$, where $\|\mathbf{L}\|$ is a majorant for the coefficients $\left|a_{j}(t)\right|, j=0,1, \ldots, n$ in $U$, and $\gamma=\gamma(K, U)$ is a constant depending only on the relative position of $K$ inside $U$, see [1, Theorem 1]. An example of computation of $\gamma(K, U)$ for a pair of concentric disks can be found below in Section 5 .

If the leading coefficient $a_{0}(t)$ vanishes somewhere in $U$, these points may still be nonsingular for a given particular solution $f$ (so called apparent singularities). The second result of [1] claims that in this case the number of real isolated zeros can grow at most polynomially in $\|\mathbf{L}\|$ (to define the $\|\mathbf{L}\|$ in this context, we normalize $\mathbf{L}$ by the condition that $\left|a_{0}(t)\right| \geqslant 1$ somewhere in $\left.K\right)$. However, in both cases the primary condition was analyticity of $f$ in a larger domain $U$ containing $K$ strictly inside.

The question about complex zeros is more subtle. The case of equations with constant coefficients was considered in [11], [12] and recently in [4], where similar results were established for bounded subdomains $K \Subset \mathbb{C}$ (note that equations with constant coefficients have a unique singularity at $t=\infty)$. In general, the number of zeros grows with the size of $K$. However, in some particular cases the estimates remain finite even for unbounded domains $K$, containing the singularity in their closure, for example, if $K$ is a horizontal strip $-\infty<a \leqslant \operatorname{Im} t \leqslant b<+\infty$ and all characteristic exponents of $\mathbf{L}$ are real.

The general problem of counting complex zeros in bounded domains for the nonsingular case was studied in [7], where it was proved that the number of zeros in this case also grows linearly in $\|\mathbf{L}\|$ even if the coefficients were variable. In [5] a sufficient condition of the form $\|\mathbf{L}\|<\varepsilon_{n}$ was found guaranteeing that any solution of $\mathbf{L} f=0$ has no more than $n-1$ roots in the unit disk, provided that the leading coefficient is identically equal to 1 .

In this paper we consider the problem of counting isolated zeros in a set $K$ containing a singular point of the operator $\mathbf{L}$ in its closure. The uniqueness theorem for analytic functions does not apply to $f$ on $K$, so the number of zeros can a priori be infinite. The asymptotical rate of accumulation of zeros to a singularity is usually studied within the framework of Nevanlinna theory [6]. On the contrary, we are interested in the cases when the number of zeros is finite, and look for explicit estimates in terms of the coefficients of $\mathbf{L}$. The simplest case is that of Fuchsian singularities. Our main result is an explicit upper bound for the number of isolated complex zeros of solutions of a Fuchsian differential equation in terms of the magnitude of coefficients of the latter. 
Besides the natural interest, our investigation was motivated by eventual applications to bifurcation of limit cycles for planar vector fields. Consider an integrable polynomial system together with a polynomial oneparametric perturbation. Then the first variation of the Poincaré first return map is an Abelian integral and satisfies the Picard-Fuchs linear differential equation which can be in many cases written explicitly, and zeros of this variation keep track of the limit cycles born out of periodic (nonisolated) orbits of the initial integrable field. The separatrix polygons correspond to singularities of both the Poincaré map and its variation; the associated Picard-Fuchs equation usually has a Fuchsian singularity with the real spectrum (for more details concerning this deeply developed subject see [2] and references therein). The results proved below allow one to majorize explicitly the number of limit cycles born by perturbation of integrable planar vector fields, as soon as the corresponding Picard-Fuchs equation is written explicitly.

1.2. Fuchsian singular points. Recall that a singular point $t=0$ of the differential operator $\mathbf{L}$ is Fuchsian, if after multiplication by an appropriate analytic function the coefficients $a_{j}(t)$ are divisible by $t^{n-j}$, for all $j=$ $0,1, \ldots, n, a_{j}(t)=t^{n-j} \widehat{a}_{j}(t)$, and $\widehat{a}_{0}(0) \neq 0$. An equivalent condition is that all solutions (in general, multivalued) grow at most polynomially at any sector with the vertex at $t=0$, see [3].

Since solutions are in general multivalued near a Fuchsian singular point, it makes sense to count their zeros in sectors with the vertex at the singularity.

Many properties of Fuchsian operators acquire a simpler form after passing to the natural logarithmic time $z=\ln t$. Suppose that the operator $\mathbf{L}$ has a unique Fuchsian singular point in the unit disk $\mathbb{D}=\{|t|<1\}$. Then this operator in the logarithmic chart can be reduced to the form

$$
\mathbf{L}=\partial^{n}+A_{1}(z) \partial^{n-1}+\cdots+A_{n-1}(z) \partial+A_{n}(z), \quad \partial=\frac{d}{d z},
$$

with $2 \pi i$-periodic coefficients $A_{j}(z)$ analytic in the left half-plane $\mathbb{H}=$ $\{\operatorname{Re} z<0\}, A_{j}(z+2 \pi i) \equiv A_{j}(z)$. The Fuchsian condition means that the coefficients $A_{j}(z)$ remain bounded in $\mathbb{H}$. Being periodic, they can be expanded in a Fourier series, hence $A_{j}(z)=A_{j}^{\circ}+O(\exp z), A_{j}^{\circ}=$ const. The linear operator $\mathbf{L}_{0}=\partial^{n}+A_{1}^{\circ} \partial^{n-1}+\cdots+A_{n-1}^{\circ} \partial+A_{n}^{\circ}, \partial=\frac{d}{d z}$, with constant coefficients, is called the principal part of $\mathbf{L}$ at infinity. Slightly abusing the language, we will refer to operators with $2 \pi i$-periodic bounded coefficients as the Fuchsian operators (resp., equations) in the half-plane.

The spectrum of the Fuchsian operator (2) is defined as the collection of roots of the characteristic polynomial $p(\lambda)=\sum_{j=0}^{n} A_{j}^{\circ} \lambda^{n-j}$ of the operator $\mathbf{L}_{0}$. 
1.3. Magnitude of the coefficients. In order to formulate the results, we introduce a one-parametric family of (essentially equivalent) numeric measures of the magnitude of coefficients of a linear operator. Below we use the notation $\mathcal{A}(U)$ for the linear space of functions analytic and bounded in $U$.

Recall that any linear differential equation $\mathbf{L} f=0$ by the standard transformation $f^{(j-1)} \mapsto Y_{j}, j=1, \ldots, n$ can be reduced to a linear system of first order differential equations $\dot{Y}=\boldsymbol{A}(z) Y, Y=\left(Y_{1}, \ldots, Y_{n}\right) \in \mathbb{C}^{n}$. Choosing any $L^{p}$-norm $\|Y\|_{p}=\left(\sum\left|Y_{j}\right|^{p}\right)^{1 / p}, p \in[1, \infty]$ on $\mathbb{C}^{n}$, we define the magnitude $\|\mathbf{L}\|_{p, U}$ of an operator $\mathbf{L}$ in a domain $U$ as the supremum $\sup _{z \in U}\|\boldsymbol{A}(z)\|_{p}$ of the associated matrix norm. In the two extreme cases $p=1$ and $p=+\infty$ this construction yields for the operator $\mathbf{L}$ with the leading coefficient $a_{0} \equiv 1$

$$
\begin{aligned}
\|\mathbf{L}\|_{1, U} & =1+\max _{j=1, \ldots, n} \sup _{z \in U}\left|a_{j}(z)\right| \\
\|\mathbf{L}\|_{\infty, U} & =\max \left\{1, \sup _{z \in U} \sum_{j=1}^{n}\left|a_{j}(z)\right|\right\} .
\end{aligned}
$$

This definition does not apply formally to operators having singular points in $U$. However, for a Fuchsian operator $\mathbf{L}$ in the unit disk $\mathbb{D}$ we define the magnitude of $\|\mathbf{L}\|_{p, \mathbb{H}}$ as the magnitude of the same operator in the logarithmic chart on $\mathbb{H}$.

Finally we introduce a mixed numeric characteristics involving both the magnitude of coefficients and the order of the equation, by putting

$$
c(\mathbf{L})=\frac{1}{2} n+19 \cdot \min _{p \in[1, \infty)}\left(\frac{1}{p} \ln n+4 \pi\|\mathbf{L}\|_{p, \mathbb{H}}\right) .
$$

The constant 19 looking somewhat mysterious outside context, is determined by the geometry of the semi-strip $\Pi$ introduced below. The value of $c(\mathbf{L})$ is in fact an upper bound for variation of argument of any solution of the equation $\mathbf{L} f=0$ along any segment of length $2 \pi$ contained in the half-plane $\mathbb{H}_{-4 \pi}=\{\operatorname{Re} z \leqslant-4 \pi\}$, see Lemma 1 .

1.4. Formulation of the main result. Let $\Pi=\{|\operatorname{Im} z| \leqslant 2 \pi$, $\operatorname{Re} z \leqslant$ $-4 \pi\}$ be the infinite horizontal semi-strip centered around the negative semi-axis and $\mathbf{L}$ a Fuchsian operator in the half-plane $\mathbb{H}$.

Theorem 1. If the spectrum of $\mathbf{L}$ is real, then any solution $f$ of the linear equation $\mathbf{L} f=0$, real on the negative semi-axis, has no more than $n(2 c(\mathbf{L})+1)$ complex isolated zeros in the semi-strip $\Pi$, where the constant $c(\mathbf{L})$ is given by (4). 
As an obvious corollary, this result gives an upper bound for the number of zeros of real solutions near a "true" Fuchsian singular point with the real spectrum in the self-overlapping sector $\{0<|t|<\exp (-4 \pi),|\operatorname{Arg} t| \leqslant$ $2 \pi\}$.

The choice of the specific semi-strip $\Pi$ is not a restriction: any other horizontal semi-strip on a positive distance from the boundary of $\mathbb{H}$ can be put into the standard position by rescaling and translations (however, such operators may affect $\|\mathbf{L}\|)$.

The assumption that the solution is real, can be replaced by the assumption that the equation is real, as the second result shows.

Theorem 2. If all coefficients of the Fuchsian operator $\mathbf{L}$ with the real spectrum are real on the real axis, then any (not necessarily real) solution may have no more than $(n+1)(2 c(\mathbf{L})+1)$ complex roots in the semi-strip $\Pi$.

Remark. One cannot remove from the assumptions of the theorems the condition that the spectrum is real, as the example of the function $f(t)=$ $t^{i}+t^{-i}=2 i \sin (\ln t)$ shows (in this case the spectrum is $\pm i$ ). Even more grave problems occur in the non-Fuchsian case.

Remark. The assumption of Theorem 2 that the coefficients of $\mathbf{L}$ are real on the real axis, formally is not restrictive. Indeed, for any Fuchsian operator with bounded coefficients one can find another Fuchsian operator $\mathbf{L}^{+}$of order not exceeding $2 n$ with real coefficients, such that any solution of the equation $\mathbf{L} f=0$ will satisfy $\mathbf{L}^{+} f=0$. This procedure is constructive (the coefficients of $\mathbf{L}^{+}$can be obtained by algebraic operations and differentiation from the coefficients of $\mathbf{L}$ ), see [3]. In particular, for each explicitly given operator $\mathbf{L}$ its "real envelope" $\mathbf{L}^{+}$can be explicitly computed. However, in general it is not possible to control the magnitude of coefficients of $\mathbf{L}^{+}$through that of $\mathbf{L}$. Besides, it is rather unnatural to require the reality of the spectrum for a totally non-real equation.

\section{Variation of argument versus growth of modulus}

The main technical result used in the proof of the theorems is an upper bound for variation of argument of an analytic function solving a linear differential equation. 
2.1. Voorhoeve index. Let $\Gamma \subset U$ be a piecewise smooth curve.

Definition 1. The Voorhoeve index of a function $f$ analytic in a neighborhood of $\Gamma$ is the total variation of argument of $f(z)$ along that curve:

$$
V_{\Gamma}(f)=\frac{1}{2 \pi} \operatorname{Var}_{\Gamma} \operatorname{Arg} f=\frac{1}{2 \pi} \int_{\Gamma}\left|\operatorname{Im}\left(f^{\prime}(z) / f(z)\right)\right||d z|
$$

(strictly speaking, this definition is valid for functions without zeros on $\Gamma$, but the general case can be studied by small perturbations).

2.2. Elementary properties. The following elementary properties of the index $V_{\Gamma}(\cdot)$ can be established, see $[11,12]$.

- The triangle inequality: if $f, g$ are two functions analytic on $\Gamma$, then

$$
\left|V_{\Gamma}(f)-V_{\Gamma}(g)\right| \leqslant V_{\Gamma}(f g) \leqslant V_{\Gamma}(f)+V_{\Gamma}(g) .
$$

- Rolle inequality for analytic functions: if $\Gamma$ is a line segment, then

$$
V_{\Gamma}(f) \leqslant V_{\Gamma}\left(f^{\prime}\right)+\frac{1}{2} .
$$

The inequality (7) is in fact a particular case of a more general inequality $V_{\Gamma}(f) \leqslant V_{\Gamma}\left(f^{\prime}\right)+(2 \pi)^{-1} \varkappa(\Gamma)$ valid for any closed curve $\Gamma$ with the integral curvature $\varkappa(\Gamma)$, see [4].

2.3. Voorhoeve index and differential equations. The index $V_{\Gamma}$ is not the only quantity satisfying the triangle and Rolle-type inequalities: in [7] it was shown that a certain characteristic expressed in terms of growth of modulus of an analytic function, called the Bernstein index, also possesses similar properties. This index can be relatively easily computed for a function satisfying a linear differential equation with bounded coefficients (since the growth of modulus can be explicitly controlled in this case), see also [1]. On the other hand, in [4] a general statement was proved asserting that the Bernstein and Voorhoeve indices are essentially equivalent in the sense that knowing one of them allows for explicit majoration of the other. In particular, the variation of argument of a function satisfying an equation $\mathbf{L} f=0$ in some domain $U \subset \mathbb{C}$ along any curve $\Gamma \Subset U$ can be explicitly estimated in terms of $\|\mathbf{L}\|_{p, U}$ and the geometry of the pair $(\Gamma, U)$.

However, in order to make the exposition self-contained, we reproduce the arguments from [4] to prove by elementary means in Section 5 the following result:

Lemma 1. Let $\mathbf{L}$ be a differential operator with coefficients analytic and bounded in the disk $4 \pi \mathbb{D}$. Then for any solution $f$ of the differential equation $\mathbf{L} f=0$ the variation of argument of $f$ along the segment $2 \pi \mathbb{I}=$ 
$[-2 \pi, 2 \pi] \Subset 4 \pi \mathbb{D}$ admits an upper estimate of the form $V_{2 \pi \mathbb{I}}(f) \leqslant c(\mathbf{L})$, where $c(\mathbf{L})$ is the number defined by (4).

This result already allows one to place explicit upper bounds on the number of complex zeros of any solution of $\mathbf{L} f=0$ in any bounded domain, thus yielding an alternative proof of one of the results from [7]. However, we need to analyze the case of the infinite semi-strip $\Pi$, and the condition of the real spectrum becomes crucial.

\section{The Petrov operator and its properties}

3.1. The Floquet operator. Denote by $\mathcal{M}$ the linear space of functions analytic in $\mathbb{H}$ and moderately growing at infinity: $f \in \mathcal{M} \Longleftrightarrow \ln |f(z)|=$ $O(|\operatorname{Re} z|)$. By $\mathcal{M}_{+} \subset \mathcal{M}$ we denote the subspace of functions real on the real axis $\mathbb{R} \subset \mathbb{C}$. Let $\mathcal{P} \subsetneq \mathcal{M}$ be the linear space (and a commutative ring) of bounded $2 \pi i$-periodic analytic functions in $\mathbb{H}: f(z) \in \mathcal{P} \Longleftrightarrow g(t)=$ $f(\ln t) \in \mathcal{A}(\mathbb{D})$.

Consider a Fuchsian operator $\mathbf{L}$ in the left half-plane $\mathbb{H}$ with $2 \pi i$ periodic coefficients $A_{0}(z) \equiv 1, A_{1}(z), \ldots, A_{n}(z) \in \mathcal{P}$ and the principal part $\mathbf{L}_{0}=\sum_{j=0}^{n} A_{j}^{\circ} \partial^{n-j}, A_{j}^{\circ}=A_{j}(-\infty)$. Denote by $\mathfrak{Z}(\mathbf{L})$ the linear space of solutions of the equation $\mathbf{L} f=0$. Clearly, $\mathfrak{Z}(\mathbf{L}) \subset \mathcal{M}$, since $\mathbf{L}$ is Fuchsian. The monodromy, or Floquet operator $\mathbf{T}: f(z) \mapsto f(z+2 \pi i)$ leaves the space $\mathfrak{Z}(\mathbf{L})$ invariant and acts identically on $\mathcal{P}$.

\section{Lemma 2 (The Frobenius theorem in the logarithmic chart).}

$$
\mathfrak{Z}(\mathbf{L}) \subset \mathcal{P} \otimes \mathfrak{Z}\left(\mathbf{L}_{0}\right) .
$$

In other words, any solution of the equation $\mathbf{L} f=0$ can be represented as a combination $\sum_{j=1}^{n} h_{j}(z) F_{j}(z)$, where $h_{j} \in \mathcal{P}$ and $F_{j} \in \mathfrak{Z}\left(\mathbf{L}_{0}\right)$.

Proof. This is a reformulation of the well-known fact [3] about representability of Fuchsian singularities in the form $\sum_{k, \lambda} h_{k, \lambda}(t) t^{\lambda} \ln ^{k} t$ with analytic coefficients $h_{k, \lambda}(t)$ (Frobenius theorem), written in the logarithmic chart.

3.2. Construction of the Petrov operator. Consider the automorphisms $\mathbf{M}_{\lambda}$ of multiplication by $\exp \lambda z: \mathbf{M}_{\lambda} f=f \exp \lambda z$ and define a parametric family of difference operators $\mathbf{P}_{\lambda}, \lambda \in \mathbb{C}$, as follows:

$$
\begin{aligned}
\mathbf{P}_{0} & =i\left(\mathbf{T}-\mathbf{T}^{-1}\right) \\
\mathbf{P}_{\lambda} & =\mathbf{M}_{\lambda} \mathbf{P}_{0} \mathbf{M}_{\lambda}^{-1}=i\left[\exp (-2 \pi i \lambda) \mathbf{T}-\exp (2 \pi i \lambda) \mathbf{T}^{-1}\right], \\
\left(\mathbf{P}_{0} f\right)(z) & =i[f(z+2 \pi i)-f(z-2 \pi i)], \\
\left(\mathbf{P}_{\lambda} f\right)(z) & =i[f(z+2 \pi i) \exp (-2 \pi i \lambda)-f(z-2 \pi i) \exp 2 \pi i \lambda] .
\end{aligned}
$$


Lemma 3. Each operator $\mathbf{P}_{\lambda}$ is a linear automorphism of $\mathcal{M}$, preserving the null space $\mathfrak{Z}(\mathbf{L})$ and commuting with multiplication by functions from $\mathcal{P}$.

If $\lambda \in \mathbb{R}$, then $\mathbf{P}_{\lambda}$ leaves invariant the subspace $\mathcal{M}_{+}$of real functions.

If $f \in \mathcal{M}_{+}$and $\mathbf{P}_{0} f=0$, then $f$ is $2 \pi i$-periodic.

Proof. The first assertion follows from the fact that both automorphisms $\mathbf{T}, \mathbf{T}^{-1}$ preserve $\mathfrak{Z}(\mathbf{L})$ and are identical on $\mathcal{P}$.

The second assertion follows from the fact that for $f \in \mathcal{M}_{+}$the functions $\mathbf{T} f$ and $\mathbf{T}^{-1} f$ are complex conjugate on the real axis $\mathbb{R}$.

3.3. Link to linear operators with constant coefficients. Any linear operator $\mathbf{L}_{0}$ with constant coefficients admits "factorization" by commuting terms (repetitions allowed)

$$
\mathbf{L}_{0}=\mathbf{D}_{\lambda_{1}} \mathbf{D}_{\lambda_{2}} \cdots \mathbf{D}_{\lambda_{n}}, \quad \mathbf{D}_{\lambda_{j}}=\mathbf{M}_{\lambda_{j}} \partial \mathbf{M}_{\lambda_{j}}^{-1}=\partial-\lambda_{j},
$$

where $\lambda_{1}, \ldots, \lambda_{n}$ are points of the spectrum of $\mathbf{L}_{0}$.

Let $\mathbf{L}$ be a Fuchsian operator of the form (2) with the principal part $\mathbf{L}_{0}$ and the spectrum $\Lambda=\left\{\lambda_{1}, \ldots, \lambda_{n}\right\}$ ordered arbitrarily. Consider the composition

$$
\mathbf{P}_{\mathbf{L}}=\mathbf{P}_{\lambda_{1}} \mathbf{P}_{\lambda_{2}} \cdots \mathbf{P}_{\lambda_{n}}
$$

defined (uniquely modulo the order of terms) by the principal part $\mathbf{L}_{0}$.

Lemma 4. Independently of the order of terms in the composition (9), the operator $\mathbf{P}_{\mathbf{L}}$ vanishes on both null spaces $\mathfrak{Z}\left(\mathbf{L}_{0}\right)$ and $\mathfrak{Z}(\mathbf{L})$.

Proof. The null space of $\mathbf{L}_{0}$ is the direct sum of nil-subspaces of $\mathbf{D}_{\lambda_{j}}$, and each $\mathbf{P}_{\lambda}$ leaves all these subspaces invariant. If $\lambda=0$ is a root of multiplicity $\nu$, then $\mathfrak{Z}\left(\mathbf{L}_{0}\right)$ contains the space of polynomials of degree $\leqslant$ $\nu-1$ as a subspace, and $\mathbf{P}_{0}^{\nu}$ vanishes on this subspace, being the difference operator. In the same way $\mathbf{P}_{\lambda}^{\nu}$ vanishes on the nil-subspace of $\mathbf{D}_{\lambda}$, thus the assertion of Lemma is proved for $\mathfrak{Z}\left(\mathbf{L}_{0}\right)$.

Any function from $\mathfrak{Z}(\mathbf{L})$ can be represented as in Lemma 2, and by the first assertion of Lemma $3, \mathbf{P}_{\mathbf{L}}\left(\sum h_{j} F_{j}\right)=\sum h_{j} \cdot \mathbf{P}_{\mathbf{L}} F_{j}=0$.

\section{Rolle theorem for Petrov operators and proof of Theorem 1}

4.1. Rolle-type inequality. Let $\Pi$ be the semi-strip from Theorem 1 and $\mathfrak{Z}_{+}(\mathbf{L})=\mathcal{M}_{+} \cap \mathfrak{Z}(\mathbf{L})$ the real subspace of the null space of a Fuchsian operator $\mathbf{L}$. For any function $f \in \mathcal{M}$ we denote by $N_{\Pi}(f)$ the number of its isolated zeros in the semi-strip $\Pi$, counted with their multiplicities. 
Lemma 5 (Rolle theorem for Petrov operators). If $\lambda \in \mathbb{R}$, then for any real function $f \in \mathfrak{Z}_{+}(\mathbf{L}) N_{\Pi}(f) \leqslant N_{\Pi}\left(\mathbf{P}_{\lambda} f\right)+2 c(\mathbf{L})+1$, where $c(\mathbf{L})$ is given by (4).

Proof. We start with the simplest case, assuming that $f$ has no zeros on the horizontal boundaries of the strip and $\lambda=0$. Consider an arbitrary rectangle $\Pi_{s}=\Pi \cap\{\operatorname{Re} z \geqslant-s\}, s<\infty$. Then by the argument principle, the number of zeros of $f$ in $\Pi_{s}$ is equal to the increment of $(2 \pi)^{-1} \operatorname{Arg} f$ along the boundary of $\Pi_{s}$ (in this case all zeros are isolated, since $f \not \equiv 0$ ). The increment of argument along the vertical sides of $\Pi_{s}$ does not exceed the total variation $V(f)$ along these sides, which is already estimated by $c(\mathbf{L})=n / 2+19\left(\frac{1}{p} \ln n+4 \pi\|\mathbf{L}\|_{p}\right)$ for each side (Lemma 1$)$. The problem is to place an upper bound for the increment of $\operatorname{Arg} f$ along the horizontal sides $H_{s}^{ \pm}=\{\operatorname{Im} z= \pm 2 \pi,-s \leqslant \operatorname{Re} z \leqslant-4 \pi\}$ of the rectangle $\Pi_{s}$.

Following [8], we observe that if $\operatorname{Im} f$ preserves its sign on a certain segment, then the increment of $\operatorname{Arg} f$ along this segment is less or equal to $\pi$. Therefore the number of zeros of the imaginary part $\operatorname{Im} f(z)$ along $H_{s}^{ \pm}$majorizes the increment $i_{ \pm}(f)=\operatorname{Arg} f(-4 \pi \pm 2 \pi i)-\operatorname{Arg} f(-s \pm 2 \pi i)$ along $H_{s}^{ \pm}:\left|i_{ \pm}(f)\right| \leqslant \pi N_{H_{s}^{ \pm}}(\operatorname{Im} f)+\pi$.

It remains only to observe that $\operatorname{Im} f(x \pm 2 \pi i)=\mp \frac{1}{2}\left(\mathbf{P}_{0} f\right)(x)$ for $x \in \mathbb{R}$, if $f$ is real on $\mathbb{R}$ (indeed, in this case $f(x+2 \pi i)=\overline{f(x-2 \pi i)}$ by the symmetry principle). Since the number of zeros of $\mathbf{P}_{0} f$ in the whole rectangle $\Pi_{s}$ is not smaller than on the real segment $\Pi_{s} \cap \mathbb{R}$, i.e., $N_{H_{s}^{ \pm}}(\operatorname{Im} f)=$ $N_{\Pi_{s} \cap \mathbb{R}}\left(\mathbf{P}_{0} f\right) \leqslant N_{\Pi_{s}}\left(\mathbf{P}_{0} f\right)$, we arrive at the conclusion

$$
N_{\Pi_{s}}(f) \leqslant 2 c(\mathbf{L})+\frac{1}{2 \pi}\left(\left|i_{+}(f)\right|+\left|i_{-}(f)\right|\right) \leqslant 2 c(\mathbf{L})+N_{\Pi_{s}}\left(\mathbf{P}_{0} f\right)+1,
$$

and since $s$ may be chosen as large as necessary, the same inequality holds for $\Pi$, as asserted by the Lemma.

If $f$ is allowed to have isolated zeros on $\{\operatorname{Im} z= \pm 2 \pi\}$ but $\operatorname{Im} f \not \equiv 0$ on these lines, then one can replace $\Pi$ by a slightly bigger semi-strip $|\operatorname{Im} z| \leqslant$ $2 \pi+\varepsilon, \operatorname{Re} z \leqslant-4 \pi$, where $\varepsilon>0$ is chosen so that $f$ has no zeros on the boundary of that bigger semi-strip. Then the above argument can be applied, and to achieve the proof in this case it remains only to note that the number of zeros of $\operatorname{Im} f(x+2 \pi i+i \varepsilon)$ for $x \in[-s,-4 \pi]$ is a semi-continuous function of $\varepsilon$.

If $\mathbb{R} \ni \lambda \neq 0$ then in order to estimate the increment of $\operatorname{Arg} f$ along the horizontal sides, one needs to apply the same considerations to the function $f(z) \exp (-\lambda z)$ which is also real on $\mathbb{R}$; the contribution along the vertical sides is estimated in the same way as before.

It remains to consider only the case of $\mathbf{P}_{\lambda} f \equiv 0$. But in this case $f(x+2 \pi i)=f(x-2 \pi i) \exp 4 \pi i \lambda$, hence the increments $i_{ \pm}(f)$ of $\operatorname{Arg} f$ along the two horizontal sides of $\Pi$ cancel each other, so that $N_{\Pi}(f) \leqslant$ 
$2 c(\mathbf{L})+0=2 c(\mathbf{L})+N_{\Pi}(0)$, as asserted by the Lemma. This last remark completes the proof.

Remark. The variation of argument of $f$ along the distant side $\{\operatorname{Re} z=$ $-s\}$ of the rectangle $\Pi_{s}$ in fact does not exceed $n \pi+\varepsilon$, if $s$ is sufficiently large. This observation allows one to improve slightly the estimate in Lemma 5 .

Remark. The formula (7) generalizes the Rolle inequality comparing the number of zeros of a real function with that of its derivative on a segment. In the same way the main result of Lemma 5 is a modification of the Rolle inequality but for difference rather for the differential operator. For more examples of Rolle-type inequalities see [4,7] and [9, section 5].

4.2. Proof of Theorem 1. Let $\mathbf{L}_{0}$ be the principal part of the operator $\mathbf{L}$ and $\mathbf{P}=\mathbf{P}_{\mathbf{L}}=\mathbf{P}_{\lambda_{1}} \cdots \mathbf{P}_{\lambda_{n}}$ the associated Petrov operator. By Lemma 4, $\mathbf{P}$ annulates any real solution $f \in \mathfrak{Z}_{+}(\mathbf{L})$. Applying $n$ times the Rolle inequality established in Lemma 5 , we arrive to the inequality $N_{\Pi}(f) \leqslant$ $n(2 c(\mathbf{L})+1)+N_{\Pi}(0)=n(2 c(\mathbf{L})+1)$. This is the upper bound we were looking for.

4.3. Proof of Theorem 2. This theorem is in fact a corollary to Theorem 1. Indeed, if $\mathbf{L}^{+}$is a real operator and $f \in \mathfrak{Z}\left(\mathbf{L}^{+}\right)$, then both $\left.\operatorname{Re} f\right|_{\mathbb{R}}$ and $\left.\operatorname{Im} f\right|_{\mathbb{R}}$ satisfy the same equation $\mathbf{L}^{+} f=0$ on $\mathbb{R}$, hence on the translates $\mathbb{R} \pm 2 \pi i$. Applying the argument principle to $f$ in $\Pi_{s}$ as in the proof of Lemma 5, we observe that

- the increment of $\operatorname{Arg} f$ along horizontal sides of $\Pi_{s}$ is less or equal to one half of the number of zeros of $\operatorname{Re} f$ plus $\frac{1}{2}$ as in the proof of Lemma 5,

- the increment of $\operatorname{Arg} f$ along the vertical sides of $\Pi_{s}$ is majorized by $2 c(\mathbf{L})$,

- Re $f$ is a real solution, hence the estimate of Theorem 1 applies.

Therefore if $\operatorname{Re} f \not \equiv 0$, then the number of zeros of $f$ in $\Pi_{s}$ is majorized by $2 \cdot \frac{1}{2}[n(2 c(\mathbf{L})+1)+1]+2 c(\mathbf{L})$. If $\operatorname{Re} f \equiv 0$, then if is already a real solution.

\section{Appendix: demonstration of Lemma 1}

5.1. Denote by $\|f(t)\|_{p}$ the norm $\left(|f(t)|^{p}+\left|f^{\prime}(t)\right|^{p}+\cdots+\left|f^{(n-1)}(t)\right|^{p}\right)^{1 / p}$. Then by the Gronwall inequality $\|Y(t)\| \leqslant\|Y(0)\| \exp \int_{0}^{t}\|A(t)\||d t|$ applied to the system of first order equations introduced in Section 1.3, we can control the growth of $\|f(t)\|_{p}$, proving for all $t \in 4 \pi \mathbb{D}$ the inequality $\|f(t)\|_{p} \leqslant\|f(0)\|_{p} \exp 4 \pi\|\mathbf{L}\|_{p}$ for any solution $f \in \mathfrak{Z}(\mathbf{L})$, where $\|\mathbf{L}\|_{p}$ is defined by (3). 
For any vector $Y=\left(y_{1}, \ldots, y_{n}\right) \in \mathbb{C}^{n}$ we have the inequalities

$$
\forall j=1, \ldots, n: \quad\left|y_{j}\right| \leqslant\|Y\|_{p}, \quad \exists j \in\{1, \ldots, n\}: \quad\left|y_{j}\right| \geqslant n^{-1 / p}\|Y\|_{p} .
$$

Therefore for one of the derivatives $g(t)=f^{(\nu)}(t)$ of some order $\nu$ between 0 and $n-1$, we have $|g(0)| \geqslant n^{-1 / p}|| f(0) \|_{p}$, and hence $|g(t)| \leqslant|g(0)| \exp B_{p}$ on $4 \pi \mathbb{D}$, where $B_{p}=\frac{1}{p} \ln n+4 \pi\|\mathbf{L}\|_{p}$. The number $B_{p}$ is a majorant for the Bernstein index of $g$, the latter in this particular case being defined as $\ln \max _{|t|=1}|g(t)|-\ln |g(0)|$ (cf. [4, 7]). Using the ideas of [4], we estimate the index $V_{2 \pi \mathbb{I}}(g)$ and then use the inequality (5) to derive the similar estimate for $f$.

5.2. By the Jensen inequality [1], [10] the function $g(t)$ has no more than $d=\left\lfloor\gamma B_{p}\right\rfloor$ isolated zeros in the disk $3 \pi \mathbb{D}$, where $\gamma=1 / \ln \frac{4}{3}$. Indeed, for the analytic function $F(z)=g(4 z)$ in the unit disk $\{|z| \leqslant 1\}$ one has the equality

$$
\ln |F(0)|=\frac{1}{2 \pi} \int_{0}^{2 \pi} \ln \left|F\left(e^{i \phi}\right)\right| d \phi+\sum_{F\left(z_{j}\right)=0} \ln \left|z_{j}\right|,
$$

with the summation extended over all roots of $F$, assuming that $F$ is nonzero at $z=0$ and on the boundary $|z|=1$. This gives the required upper bound for the number of zeros of $F(z)$ (resp., $g(t)$ ) inside the disk $\{|z| \leqslant 3 / 4\}$ (resp., $\{|t| \leqslant 3 \pi\})$.

5.3. Consider the ratio $h(t)=g(t) / P(t), P(t)=\left(t-t_{1}\right) \cdots\left(t-t_{d}\right)$, where $t_{j}$ are roots of $g$ in $3 \pi \mathbb{D}$ (counted with their multiplicities). Due to the prescribed location of the roots $t_{j}$, any linear factor $\left|t-t_{j}\right|$ is greater or equal to $\pi$ on the boundary of $4 \pi \mathbb{D}$ and less or equal to $3 \pi$ at the origin. Therefore $|P(0)|=\prod_{j=1}^{d}\left|t_{j}\right| \leqslant(3 \pi)^{d}$, and $|P(t)| \geqslant \pi^{d}$ on the boundary $|t|=4 \pi$, so finally we conclude that $|h(t)| \leqslant|h(0)| \cdot 3^{d} \exp B_{p}$ everywhere in $4 \pi \mathbb{D}$ and, moreover, in $3 \pi \mathbb{D}$ the function $h(t)$ is nonvanishing.

5.4. Without loss of generality we may assume that $h(0)=1$. Choose a branch of logarithm $\xi(t)=\ln h(t)$ for $t \in 3 \pi \mathbb{D}$ in such a way that $\xi(0)=0$. Then $\xi(\cdot)$ maps the disk $3 \pi \mathbb{D}$ into the translated left half-plane $\mathbb{H}_{b}=\{\operatorname{Re} \xi \leqslant c\}$, where $b=d \ln 3+B_{p}=(1+\gamma \ln 3) B_{p}$.

Let $\Psi: \mathbb{D} \rightarrow \mathbb{H}_{1}=\{\operatorname{Re} z<1\}, \Psi(t)=2 t /(t+1)$, be the standard map taking $t=0$ into the origin and mapping the boundary of the unit disk into the boundary $\operatorname{Re} z=1$. By the Shwartz lemma, for any other map $\psi: \mathbb{D} \rightarrow \mathbb{H}_{1}$ taking 0 into 0 , the image of the disk $\psi(r \mathbb{D}), 0<r<1$, is entirely contained in $\Psi(r \mathbb{D})$, in particular, $|\psi(t)| \leqslant 2 r /(1-r)=|\Psi(-r)|$ for $|t| \leqslant r$. Applying this observation to the function $\psi(t)=b^{-1} \xi(t / 3)$, we arrive to the inequality $|\xi(t)| \leqslant 10 b$ for $t \in \frac{5}{2} \pi \mathbb{D}$. An alternative 
way to prove this upper bound is to apply the Harnack inequality to the nonnegative harmonic function $u(z)=\left(d \ln 3+B_{p}\right) \ln |h(0)|-\ln |h(z)|$.

5.5. Consider a part of diameter $K=[-a, a] \Subset \mathbb{D}$ of the unit disk, and let $F: \mathbb{D} \rightarrow \mathbb{D}$ be an analytic mapping of $\mathbb{D}$ into itself. Then for the length of the image $|F(K)|$ we have the following inequality for the length $|F(K)|$ of the image $F(K)$, obtained via the Cauchy integral formula:

$$
\begin{aligned}
|F(K)| & \leqslant \int_{-a}^{a}\left|F^{\prime}(x)\right| d x=\int_{-a}^{a} d x \frac{1}{2 \pi} \oint_{\partial \mathbb{D}} \frac{|F(z)|}{|z-x|^{2}}|d z| \\
& =\int_{-a}^{a} \frac{d x}{2 \pi} \int_{0}^{2 \pi} \frac{d \phi}{1+x^{2}-2 x \cos \phi}=\int_{-a}^{a} \frac{d x}{1-x^{2}}=\left.\frac{1}{2} \ln \frac{1+x}{1-x}\right|_{-a} ^{a} \\
& =\ln \frac{1+a}{1-a} .
\end{aligned}
$$

After obvious rescaling arguments, this implies the inequality $|\xi(2 \pi \mathbb{I})| \leqslant$ $\ln 9 \cdot 10 b$. The variation of argument of $h(\cdot)=\exp \xi(\cdot)$ along $2 \pi \mathbb{I}$ is less or equal to the length $|\xi(2 \pi \mathbb{I})|$. Finally we conclude that

$$
V_{2 \pi \mathbb{I}}(h) \leqslant 5 \pi^{-1} \ln 9 \cdot b=5 \pi^{-1}(1+\gamma \ln 3) \ln 9 \cdot B_{p} .
$$

5.6. The total variation of argument of $P(t)$ along $2 \pi \mathbb{I}$ does not exceed $\pi d$, since $\mathbb{I}$ is a straight line segment. Using the inequality (7), we can estimate the variation of argument of $f(t)$ via that of $f^{(\nu)}$ :

$$
\begin{aligned}
V_{2 \pi \mathbb{I}}(f) & \leqslant \nu / 2+V_{2 \pi \mathbb{I}}(P)+V_{2 \pi \mathbb{I}}(h) \leqslant \nu / 2+d / 2+5 \pi^{-1} b \ln 9 \\
& \leqslant n / 2+\gamma B_{p} / 2+5 \pi^{-1} \ln 9(1+\gamma \ln 3) B_{p} \\
& =n / 2+B_{p} \cdot\left\{1 /\left(2 \ln \frac{4}{3}\right)+\frac{5}{\pi} \ln 9\left[1+(\ln 3) /\left(\ln \frac{4}{3}\right)\right]\right\} \\
& \approx n / 2+18.5895 B_{p}<n / 2+19\left(\frac{1}{p} \ln n+4 \pi\|\mathbf{L}\|_{p}\right) .
\end{aligned}
$$

Remark. The constant $18,5 \cdots<19$ can be slightly improved by choosing different relative sizes of the disks, instead of the scheme $2 \pi \mathbb{D} \Subset \frac{5}{2} \pi \mathbb{D} \Subset$ $3 \pi \mathbb{D} \Subset 4 \pi \mathbb{D}$.

Moreover, the inequality asserted by Lemma 1 is subject to a certain trade-off between the term $n / 2$ and the term proportional to $\|\mathbf{L}\|_{p}$ : if the latter is substantially overtaking the former, then one could partition the segment $2 \pi \mathbb{I}$ into a number of smaller subsegments. Then the relative position of each segment in the disk $4 \pi \mathbb{D}$ will be "better", i.e., allowing for a better estimate for the variation of argument of the corresponding derivative $g=f^{(\nu)}$, but the term $n / 2$ will be replaced by $k n / 2$, where $k$ is the number of subsegments.

However, all these improvements still do not yield a sharp estimate for the number of zeros, therefore we restrict ourselves to just indicating how 
they could be made when really necessary. Our goal was only to stress the explicit nature of the estimates.

\section{Acknowledgments}

We are grateful to Askold Khovanskiu and German Petrov for the explanations concerning their unpublished work. Dmitri Novikov helped us on all stages in the preparation of this work. Many people have expressed stimulating interest in the results of this paper. Our sincere gratitude goes to all of them.

\section{References}

1. Yu. Il'yashenko and S. Yakovenko, Counting real zeros of analytic functions satisfying linear ordinary differential equations, J. Differential Equations 126 (1996), no. $1,87-105$.

2. - Double exponential estimate for the number of zeros of complete Abelian integrals, Invent. Math. 121 (1995), no. 3, 613-650.

3. E. L. Ince, Ordinary differential equations, Dover, 1956.

4. A. Khovanskiı̌ and S. Yakovenko, Generalized Rolle theorem in $\mathbb{R}^{n}$ and $\mathbb{C}, \mathrm{J}$. Dynamical and Control Systems 2 (1996), no. 1, 103-123.

5. W. J. Kim, The Schwarzian derivative and multivalence, Pacific J. of Math., 31 (1969), no. 3, 717-724.

6. I. Laine, Nevanlinna theory and complex differential equations, De Gruyter studies in mathematics, vol. 15, Walter de Gruyter, Berlin, 1993.

7. D. Novikov and S. Yakovenko, A complex analog of Rolle theorem and polynomial envelopes of irreducible differential equations in the complex domain, J. London Math. Soc. (2) (1996), to appear.

8. G. Petrov, Nonoscillation of elliptic integrals, Functional Anal. Appl. 24 (1990), no. $3,45-50$.

9. G. Pólya and G. Szegö, Aufgaben und Lehrsätze aus der Analysis, third ed., vol. 2, Springer-Verlag, Berlin e.a., 1964.

10. B. Shabat, Introduction to complex analysis, Transl. Math. Monographs, vol. 110, AMS publ., Providence, 1992.

11. M. Voorhoeve, On the oscillation of exponential polynomials, Math. Z. 151 (1976), $277-294$.

12. , A generalization of Descartes' rule, J. London Math. Soc. (2) 20 (1979), 446-456.

Department of Mathematics, Yale University, New Haven CT 06520

E-mail address: roitman@math.yale.edu

Department of Theoretical Matehmatics, Weizmann Institute of Science, REHOVOT 76100, ISRAEL

E-mail address: yakov@wisdom.weizmann.ac.il 